\title{
Decentralized Routing in Nonhomogeneous Poisson Networks
}

\author{
Chi Zhang, Pan Li, Yuguang Fang and Pramod P. Khargonekar \\ Department of Electrical and Computer Engineering \\ University of Florida, Gainesville, FL 32611 \\ Email: \{zhangchi@, lipanleo@,fang@ece.,ppk@eng.\}ufl.edu
}

\begin{abstract}
In his seminal work, Jon Kleinberg considers a small-world network model consisting of a $k$-dimensional lattice augmented with shortcuts. Under the assumption that the probability of a shortcut being present between two nodes $u$ and $v$ decays as a power, $d(u, v)^{-\alpha}$, of the distance $d(u, v)$ between them, Kleinberg shows that decentralized routing scheme such as greedy geographic routing is efficient if $\alpha=k$ and that there is no efficient decentralized routing algorithm if $\alpha \neq k$. The results are extended to a continuum model recently, wherein the nodes are distributed as a homogeneous Poisson point process by Franceschetti and Meester, Draief and Ganesh. In our work, we extend the result further to a more realistic model constructed from a nonhomogeneous Poisson point process, wherein each node is connected to all its neighbors within some fixed radius, as well as possessing random shortcuts to more distant nodes. More importantly, we show that in nonhomogeneous cases, the necessary and sufficient condition for greedy geographic routing to be efficient is that the probability of a shortcut being present from node $u$ to $v$ should be inversely proportional to the number of nodes which are closer to $u$ than $v$ is. We also demonstrate some applications of our results to wireless networks.
\end{abstract}

\section{INTRODUCTION}

The modern view of the so-called small-world phenomenon can be traced back to the famous experiments by Stanley Milgram in the 1960s [1]. His work showed that any two people in the world can be connected by a chain of (on the average) six acquaintances, and people can deliver messages efficiently to an unknown target via their acquaintances. The small-world phenomenon has also been shown to be pervasive in networks from nature and engineering systems, such as the nervous system of the nematode worm Caenorhabditis elegans [2], food webs [3], the World Wide Web [4], [5], P2P systems [6]-[8], "web of trust" for security systems [9], etc.

Graph models to explain why social networks develop a small diameter (maximum hopcount of the shortest paths), have been around for some time. While Erdös-Rényi random graphs possess the property of having a small diameter [10], [11], it is well-known that they are not good models for social networks because of the assumption of independence of links [4] $]^{1}$. Watts and Strogatz [4] conduct a set of rewiring experiments on graphs, and observe that by re-wiring a few random links in finite lattices, the average path length could be reduced drastically, which is smaller than logarithm

This work was supported in part by the U.S. National Science Foundation under grant CNS-0721744, CNS-0716450 and CNS-0626881.

${ }^{1}$ For the same reason, Erdös-Rényi random graph is also not a good model for the wireless networks, see [12] for a more detailed explanation. of the number of vertices (nodes/points) of the graph. This leads them to propose the classic discrete small-world model which essentially consists of a lattice augmented with random links acting as shortcuts.

The sociological experiments of Milgram demonstrated not only the existence of short chain of acquaintances between strangers but also the ability of people at finding such chains. Which graph models have this property? Specifically, when can decentralized routing algorithms (which we will formally define later) find a short path between arbitrary source and destination nodes? This question is first addressed by Kleinberg [13], [14] for the class of finite $k$-dimensional lattices augmented with long-range connections (shortcuts) chosen randomly from the $\alpha$-harmonic distribution, that is, a longrange link between nodes $u$ and $v$ exists with probability proportional to $d(u, v)^{-\alpha}$, where $d(u, v)$ denotes the Manhattan distance between nodes $u$ and $v$. Kleinberg shows that the simple geographic greedy routing algorithm by using only local information can route messages between any two nodes in $O\left(\log ^{2} n\right)$ expected number of hops if $\alpha=k$ and that there is no efficient decentralized routing algorithm if $\alpha \neq k$. Note that there is a fundamental difference between the existential discovery and the algorithmic discovery. It is quite possible that short paths exist, but that these cannot be found by any algorithm using only local knowledge of the network. For example, Kleinberg's results show that decentralized routing algorithms cannot find short routes when $\alpha \neq k$, even though such routes indeed exist for $\alpha<2 k$, as demonstrated in [15]. While it is a well recognized seminal contribution, Kleinberg's model is slightly unnatural since it is a discrete model that assumes all nodes to be located on a lattice, which is often not the case in the real world.

In this paper, we first extend Kleinberg's result to a more realistic model constructed from a geometric network, wherein the nodes are distributed in a 2-dimensional or 3dimensional space as a spatial Poisson point process (homogeneous or nonhomogeneous), and the probability of an edge (link/connection) between a pair of nodes $u$ and $v$ is given by a function $g(\cdot)$ of the distance $d(u, v)$, as well as the population between the nodes. Such spatial graph models and variants thereof arise, for instance, in the study of social networks or wireless communication networks. More importantly, we show that in nonhomogeneous cases, the necessary and sufficient condition for greedy geographic routing to be efficient is that the probability of a shortcut being present from node $u$ to 
$v$ should be inversely proportional to the number of nodes which are closer to $u$ than $v$ is. Note that our model gives the same shortcut probabilities as models in previous work wherein the nodes are distributed uniformly. Therefore, our work can also be applied to homogeneous cases and gives more general condition on the navigability of any geometric network. Our result shows that it is the population-density based shortcut distribution which relates to the navigability of the geometric networks rather than the geographic-distance based shortcut distribution suggested in Kleinberg's work.

\section{RELATED WORK}

\section{A. Related Work on Social Networks}

Kleinberg's seminal work introduced a new theme in the network research literature: "navigable small-world networks". Most important advances along this theme have been summarized in Kleinberg's recent address [16] at the 2006 International Congress of Mathematicians. One interesting line of research is related to the design of decentralized routing algorithm. Recall that Kleinberg shows that the simple greedy routing algorithm by using only local information can route messages between any two nodes in $O\left(\log ^{2} n\right)$ expected number of hops if $\alpha=k$. This bound is tightened to $\Theta\left(\log ^{2} n\right)$ later by Barrière et al. [17] and Martel et al. [18]. Since the expected diameter of a $k$-dimensional Kleinberg network is $\Theta(\log n)$ [18], there is still some room for improving the routing performance. Further research [7], [18]-[20] shows that in fact the $O\left(\log ^{2} n\right)$ bound of the original greedy routing algorithm can be improved by putting some extra information in each message holder, which means there are some trade-off between the routing efficiency and memory space for different decentralized routing schemes. In this paper, however, we focus on deriving the condition on the shortcut distribution which guarantees the navigability of a geometric network in a more general setting. Note that the research along this line is orthogonal and complementary to our work, in the sense that only when the geometric graph itself is navigable (which is guaranteed by our results), all those proposed more complicated geographic routing algorithms can be applied in order to further improve the routing performance.

Kleinberg's original discrete model is extended to continuum models recently, wherein the nodes are distributed as a homogeneous Poisson point process by Franceschetti and Meester [21], Draief and Ganesh [22]. The homogeneous Poisson location of people (for social networks) or the network devices (for wireless networks) reflects various irregularities of a real network architecture. This irregularity is, however, homogeneous in [21], [22], meaning that the respective mean densities are constant in the space. This assumption is often not very realistic. It is enough to take a look at a map of the density of population of a given region to realize that the social network and an optimal communication network that is supposed to reflect the traffic demand, should also be nonhomogeneous, which indicates the significance of our new model (cf. Section III) which is based on a nonhomogeneous Poisson point process of node distribution. Moreover, unlike the work of [21], [22] wherein the probability of shortcuts is solely determined by geographic distance, we adopt a different population-density based shortcut formation scheme.

The idea of adding shortcuts with probability inversely proportional to the number of closer candidates comes from Liben-Nowell et al.'s empirical investigation [23] of the real social network which comprises the 1,312,454 bloggers in the LiveJournal online community (www.livejournal.com), in February 2004. They find that Kleinberg's model cannot be used to explain the navigability of the LiveJournal network because of a large variance in population density across the space. They propose a new density-aware model of shortcut formation scheme to deal with the variance problem in population density. This idea is also implicit in Kleinberg's work on his group-structure model [24], which is based on people's membership in groups like organizations or neighborhoods. This model, a generalization of his lattice-based model [13], [14], introduces a long-range link between $u$ and $v$ with probability inversely proportional to the size of the smallest group containing both $u$ and $v$. When the groups satisfy two key properties: a member of a group $g$ must always belong to a subgroup of $g$ that is not too much smaller than $g$, and every collection of small groups with a common member must have a relatively small union, Kleinberg has proven that the resulting network is a navigable small world. The main difference between the work in [23], [24] and that of this paper is that (i) unlike previous work based on discrete settings, to the best of our knowledge, this is the first work to prove the navigability of the geometric networks with the populationdensity based shortcut formation scheme in the general or nonhomogeneous continuum setting; (ii) unlike previous work with full scale invariance, in our model the scale invariance is cut off at very small and very large distances. Therefore in our model the long-range connections for each node is $O(1)$ (cf. Section IV-A) whereas in previous work it is $O(\log n)$. Note that our model is more realistic in that maintaining long-range connections is obviously more difficult for social or hybrid wireless communication networks and should be avoided as much as possible.

\section{B. Related Work on Wireless Networks}

Small world network model is also introduced in the wireless networking scenarios. For a wireless network, communication devices (or nodes) are distributed in a 2-dimensional or 3-dimensional space, and each node is connected to all its neighbors within some fixed radius $r_{c}$, which is called the transmission range. All those connections can be treated as local connections in Kleinberg's model [13]. Gupta and Kumar [25] show that when $n$ nodes are distributed uniformly and randomly in the plane, the average number of hops along the shortest path between two randomly chosen nodes (source-destination pair) is $O(\sqrt{n})$. In mathematics, this kind of network is termed Random Geometric Graph (RGG) [26], which can be denoted as $G\left(n, r_{c}\right)$ and is obviously not a small world network. Since the forwarding burden is proportional to the average route hop-length, the hopcount on the order of $n$ is one of the determining factors that cause the achievable throughput for each source-destination pair to approach 0 as 
the network size $n$ grows to infinity [25].

In order to handle this vanishing throughput effect, many works [27]-[32] suggest adding a wired infrastructure to an unstructured (ad-hoc) wireless network. Their results show that when a small number of wired or wireless (e.g., directional antenna) long-range connections are added in the ad hoc networks, the average route hop-length can be significantly reduced, and better scaling law of throughput can be achieved.

Hybrid sensor networks with wired shortcuts have also been proposed in the literature [32]-[34]. A hybrid sensor network differs from a hybrid ad hoc network in that the communication in the hybrid sensor network is many-toone (sensor-to-sink), rather than many-to-many. Based on the specific communication type of sensor networks, some wired shortcut placement schemes have been proposed. Furthermore, their corresponding improvement in energy efficiency, which is directly related to the hop-length, has been investigated.

The common problems of those works include: they do not address the problem of how to find those short paths by utilizing augmented shortcuts. Given the nodes are distributed as a homogeneous Poisson point process, the graph is connected only with the local connections, and the shortcuts are distributed in Kleinberg's fashion, Draief and Ganesh [22] show that no decentralized routing scheme can find short paths with hopcount smaller than $O\left(n^{\gamma}\right)$ for any $\gamma<(2-\alpha) / 6$ when $\alpha<2$, even though there exists such short paths with $O(\log n)$ hops. Therefore, it is not necessary that all the improvement introduced by the infrastructure to the ad hoc networks can be achieved. In addition, most of these works assume that the nodes are distributed as a homogeneous Poisson point process, which is not realistic as mentioned before.

\section{NETWORK MODEL}

\section{A. Notation and Network Model}

We use the following notation throughout the paper:

- $f(n)=O(g(n))$ means that there exists a constant $c$ and integer $N$ such that $f(n) \leq c g(n)$ for $n>N$.

- $f(n)=\Theta(g(n))$ means that $f(n)=O(g(n))$ and $g(n)=$ $O(f(n))$.

- $f(n)=\Omega(g(n))$ means that $g(n)=O(f(n))$.

- With high probability (w.h.p.) refers to a probability at least $1-\epsilon(n)$, for a function $\epsilon(n)$ going to 0 with $n \rightarrow \infty$.

- Pr stands for probability of, and $\mathbf{E}$ is the corresponding expectation.

- $\log$ denotes the logarithm with base 2 while $\ln$ denotes the natural logarithm with base $e$.

- $d(u, v)$ means the Euclidean distance between two points $u$ and $v$, where $u, v \in \mathbb{R}^{2}$ or $\mathbb{R}^{3}$.

- ball $(u, r)$ means the closed ball of radius $r$ and centered at point $u$, i.e. $\operatorname{ball}(u, r)=\{w: d(w, u) \leq r\}$.

- $L_{u v}$ is the lune of $u$ and $v$ where $u, v \in \mathbb{R}^{2}$ or $\mathbb{R}^{3}$, i.e., $L_{u v}=\operatorname{ball}(u, d(u, v)) \cap \operatorname{ball}(v, d(u, v))$.

- $\operatorname{pop}(A)$ means the number of nodes located in the region $A$ where $A \subseteq \mathbb{R}^{2}$ or $\mathbb{R}^{3}$ is measurable.

- $|A|$ is the shorthand for 2-dimensional or 3-dimensional Lebesgue measure of a measurable set $A \subseteq \mathbb{R}^{2}$ or $\mathbb{R}^{3}$. All integrals considered will be Lebesgue integrals.
Definition 1: [Poisson Point Process] A Poisson point process $\mathcal{P}$ with density measure $\Lambda$ (a diffuse Radon measure) is a point process possessing the two following properties:

- [Poisson distribution of point-counts]: the number of points in a bounded Borel set $B$ has a Poisson distribution with mean $\Lambda(B)$, i.e.,

$\operatorname{Pr}[\mathcal{P}(B)=m]=\frac{(\Lambda(B))^{m}}{m !} \cdot \exp (-\Lambda(B))$ for $m=0,1,2, \ldots$

- [Independent scattering]: the number of points in $k$ disjoint Borel sets form $k$ independent random variables.

We assume throughout the paper that the Radon measure $\Lambda$ has a density with respect to Lebesgue measure, and it can be written as [35]

$$
\Lambda(B)=\int_{B} \lambda(x) d x \text { for Borel sets } B .
$$

The density $\lambda(x)$ is called the intensity function of the general Poisson point process.

In this work, we consider a network model constructed from a nonhomogeneous Poisson point process on a finite square $\mathcal{S}_{n}$, wherein each node is connected to all its geographic neighbors within some fixed radius, as well as possessing random shortcuts to more distant nodes. More precisely:

Definition 2: [Network Model]

- [Node distribution] We consider a sequence of graphs indexed by $n \in \mathbb{N}$. Nodes $\left\{x_{i}\right\}$ form a nonhomogeneous Poisson process defined by Def. 1, with density function $\lambda$ having connected and compact support $\Psi$ with smooth boundary $\partial \Psi$, and $\frac{\max _{\Psi} \lambda}{\min _{\Psi} \lambda} \leq c_{\lambda}$ where $c_{\lambda}$ is a constant. ${ }^{2}$

- [Local link] Each node $x_{i}$ is connected to all nodes $x_{j}$ that their Euclidean distance is not greater than $r_{n}=\sqrt{\left(c_{g} \cdot \ln n\right) / n}$ for 2-dimensional case or $r_{n}=$ $\sqrt[3]{\left(c_{g} \cdot \ln n\right) / n}$ for 3 -dimensional case. These links are referred to as local links and the corresponding nodes as the local neighbors of $x_{i}$. The graph with vertex set $V=\left\{x_{i}: 1 \leq i \leq n\right\}$ and edge set consisting solely of local links is a nonhomogeneous RGG denoted as $G\left(n, r_{n}\right)$. Parameter $c_{g}$ is a constant called GEOGREEDY parameter and will be discussed in Section IV-B.

- [Shortcut] For two nodes $x_{i}$ and $x_{j}$ such that $d\left(x_{i}, x_{j}\right)>r_{n}$, the link $\left(x_{i}, x_{j}\right)$ is present with probability $\operatorname{Pr}\left[\left(x_{i}, x_{j}\right)\right]=a_{n} \cdot\left(\operatorname{pop}\left(\operatorname{ball}\left(x_{i}, d\left(x_{i}, x_{j}\right)\right)\right)\right)^{-\alpha}$, where $a_{n} \geq 0$ and $\alpha \geq 0$ are universial constants. These links are referred to as shortcuts and the corresponding nodes as the long-range neighbors of $x_{i}$. The graph with vertex set $V$ and edge set consisting of local links and shortcuts is called a nonhomogeneous Poisson Network denoted as $\operatorname{NPN}\left(n, r_{n}, \alpha\right)$. Parameter $a_{n}$ is a function of $n$, which is called normalization parameter and will be discussed in Section IV-A.

Here, we give some intuition behind our network model. This model has a simple "geographic" interpretation for social

\footnotetext{
${ }^{2}$ We recall that the support $\Psi$ of a probability density function is the set of points in which it has nonzero value, and that the boundary $\partial \Psi$ is smooth if and only if it is twice differentiable.
} 


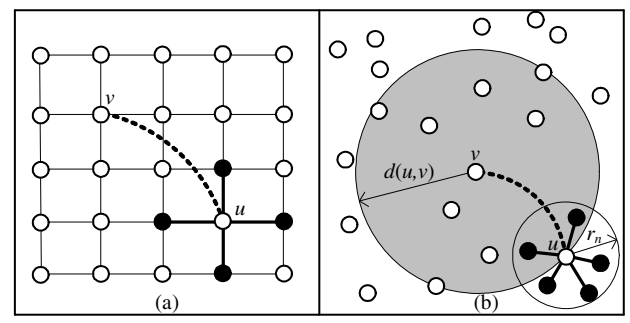

Fig. 1. Navigable small-world network models. (a) Kleinberg's augmented lattice model in [13]; (b) Nonhomogeneous Poisson network model used in this paper. Here stuffed nodes, bold solid lines and bold dashed curves represent the local neighbors of $u$, local links and shortcuts, respectively. Note that in (b), the probability of obtaining a shortcut from $u$ to $v$ is inversely proportional to the number of nodes within the shaded disk ball $(v, d(u, v))$.

networks: the nodes of a social network are the people in it; two nodes are connected if these two persons know each other. Individuals know their neighbors within some fixed small radius $r_{n}$; they also have some number of acquaintances distributed more broadly across the space. Obviously, the probability that two persons $u$ and $v$ know each other should decrease as the geographical distance between them increases. In Kleinberg's model, this probability is solely determined by the geographical distance between them (see Fig. 1 (a)), while in our model, this probability is determined by the population in ball $(v, d(u, v))$ (see Fig. 1 (b), the shaded disk). Intuitively, one justification for this kind of shortcut distribution is the following: in order to befriend $u, v$ will have to compete with all of the more "convenient" candidate friends for $u$, i.e., all people who live closer to $u$ than $v$ does. Therefore, in this paper, when we are modeling the distribution of shortcuts, we consider the population densities as well as the geographical distances. Our consideration is more reasonable since the probability of two persons know each other should decrease more quickly with the geographical distance when the population density is high. It is a well-known observation that in the city with high population density, people know their geographical neighbors with a small probability while in the country, this probability will be much higher.

For wireless ad hoc networks, local links model the communication between nearby nodes through wireless links. This kind of disk communication model (with communication range $r_{n}$ ) is widely used in the theoretical study of wireless networks (see [25] for an example). Shortcuts can model the wired or wireless infrastructure added in this purely ad hoc network. Obviously, the probability of existence of the shortcut should decrease as the geographical distance between them increases since the cost of the shortcuts (wired infrastructure) is proportional to the total length of the wires deployed [32]. The node density also need be included in the consideration in order to optimize the placement of the wired infrastructure.

\section{B. Background}

Here we present the formal definitions of the concepts used in this paper.

Definition 3: [Small-World Network] For a geometric network to be termed a small-world network (SWN), its diameter should be on the order of $\log n$ or at most polylogarithmic in $n$, where $n$ is the network size.
The objective is to route a packet from an arbitrary source node $s$ to an arbitrary destination $t$ using a small number of hops. We are interested in decentralized routing algorithms, which do not require global knowledge of the graph topology.

Definition 4: [Decentralized Routing] A decentralized routing algorithm specifies a sequence of nodes $s=$ $x_{1}, x_{2}, \ldots, x_{k}=t$ where the only requirement is that each node $x_{i}(2 \leq i \leq k-1)$ should be chosen from the local or long-range neighbors of node $x_{i-1}$, and $x_{i}$ only knows the topology of its neighbors (local information).

One special kind of decentralized routing is called geographically greedy routing or GEOGREEDY in shorthand.

Definition 5: [Geographically Greedy Routing] It is assumed that each node knows its location (coordinates) in the space, as well as the location of all its neighbors (both local and long-range), and of the destination $t$ (the header of the packet carries the location of $t$ ). If there is no direct link from the source $s$ to the destination $t$, the current packetholder $u$ will forward the packet to one of its local or longrange neighbors closest to the destination $t$. If none of these neighbors are closer to the destination of the packet than the packet-holder itself, the packet will be discarded.

Definition 6: [Navigable Small-World Network] A SWN is called navigable $S W N$ if and only if there exists decentralized routing algorithm such that the number of hops for message delivery between any pair of nodes is of order at most polylogarithmic in $n$, where $n$ is the network size.

\section{Characterization of the Parameters in the NETWORK MODEL}

There are two parameters $c_{g}$ and $a_{n}$ in our network model left to be determined in Section III. In this section, we will show how to tune these two parameters in order to obtain the network model with the desired properties.

\section{A. Normalization Parameter $a_{n}$ and the Expected Number of Shortcuts for Each Node}

Since the maintenance of shortcuts are costly both in social and wireless networks, in our model we upper-bound the expected number of shortcuts for each node as a constant number $\Theta(1)$.

For any node $v \in V$, we have:

$$
\begin{aligned}
\sum_{x_{i} \in V, x_{i} \neq v} \operatorname{Pr}\left[\left(v, x_{i}\right)\right] & =a_{n} \cdot\left(\sum_{i=1}^{n-1} \frac{1}{i^{\alpha}}\right) \\
& = \begin{cases}a_{n} \cdot \Theta\left(\frac{1}{1-\alpha} n^{1-\alpha}\right) & \text { if } \alpha<1 \\
a_{n} \cdot \Theta(\ln n) & \text { if } \alpha=1 \\
a_{n} \cdot \Theta\left(\frac{1}{\alpha-1}\right) & \text { if } \alpha>1\end{cases}
\end{aligned}
$$

Therefore, if we require the expected number of shortcuts for each node, i.e., $\sum_{x_{i}} \operatorname{Pr}\left[\left(v, x_{i}\right)\right]$, to be a constant number $\Theta(1)$, we need to have:

$$
a_{n}= \begin{cases}\Theta\left((1-\alpha) \cdot n^{\alpha-1}\right) & \text { if } \alpha<1 \\ \Theta(1 / \ln n) & \text { if } \alpha=1 \\ \Theta(\alpha-1) & \text { if } \alpha>1\end{cases}
$$




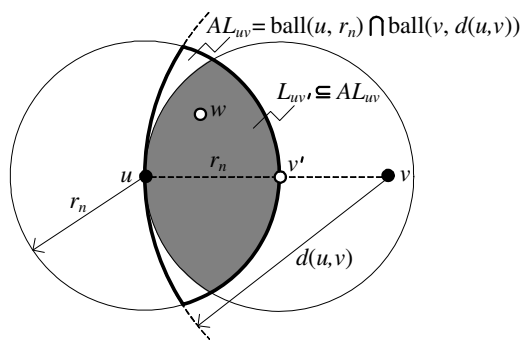

Fig. 2. Sufficient condition for $u$ always having a local neighbor $w$ closer to the destination $v$ with $d(u, v)>r_{n}$. Note that $v^{\prime}$ is the intersection point of the segment $u v$ and the circulus of ball $\left(u, r_{n}\right)$. The shaded area is $L_{u v^{\prime}}$ which is contained in $A L_{u v}$ (the area which is circulated by the bold curves).

\section{B. GeOGREedy Parameter $c_{g}$ and the Expected Number of Local Neighbors for Each Node}

We will implicitly assume that any node can find a local neighbor closer to the destination $t$ than itself in the following discussion (cf. Section V), and call it the successful GEOGREEDY assumption. This assumption implies that, GEOGREEDY algorithm can successfully route packets between any source-destination pairs from $V \times V$ on the network $G\left(n, r_{n}\right)$. We first show that this assumption holds w.h.p. if $c_{g}$ is chosen to be sufficiently large.

Theorem 1: Given the network model $N P N\left(n, r_{n}, \alpha\right)$ (cf. Def. 2) and the randomly chosen destination $t$, the sufficient condition for any node to find a local neighbor closer to $t$ than itself w.h.p. is that

$$
\begin{gathered}
c_{g}>\frac{6}{(4 \pi-3 \sqrt{3}) \cdot \min _{\Psi} \lambda} \approx \frac{0.82}{\min _{\Psi} \lambda} \text { for 2-D case; } \\
\quad \text { or } c_{g}>\frac{12}{5 \pi \cdot \min _{\Psi} \lambda} \approx \frac{0.77}{\min _{\Psi} \lambda} \text { for 3-D case. }
\end{gathered}
$$

Proof: For the 2-dimensional case, a straightforward calculation yields that

$$
\left|L_{u v}\right|=\left(\frac{2 \pi}{3}-\frac{\sqrt{3}}{2}\right) \cdot(d(u, v))^{2},
$$

while for the 3-dimensional case, we obtain

$$
\left|L_{u v}\right|=\frac{5 \pi}{12} \cdot(d(u, v))^{3} .
$$

We set $\delta=\frac{2 \pi}{3}-\frac{\sqrt{3}}{2}$ and focus on the 2-dimensional case in the following. The proof for the 3-dimensional case is almost the same except for setting $\delta=\frac{5 \pi}{12}$.

Two key observations from Fig.2 are:

(i) The sufficient and necessary condition that node $u$ can find a local neighbor closer to the destination $v$ with $d(u, v)>r_{n}$ is that there exists at least one node $w \in V$ within $A L_{u v}$, where $A L_{u v}$ is defined as $A L_{u v}=\operatorname{ball}\left(u, r_{n}\right) \cap$ $\operatorname{ball}(v, d(u, v))$.

(ii) $L_{u v^{\prime}} \subseteq A L_{u v}$ where $v^{\prime}$ is the intersection point of the segment $u v$ and the circulus of $\operatorname{ball}\left(u, r_{n}\right)$.

Therefore, the sufficient condition that node $u$ can find a local neighbor closer to the destination $v$ is that there exists at least one node $w \in V$ within $L_{u v^{\prime}}$. From eq. (2), we obtain

$$
\left|L_{u v^{\prime}}\right|=\delta \cdot r_{n}^{2}
$$

Let $X_{i}$ be the event that node $x_{i}$ does not have any local neighbors closer to the destination, and let $X=\bigcup_{i=1}^{n} X_{i}$ be the event that there is at least one node in $V$ which does not have any local neighbors closer to the destination. From eq. (4), we obtain

$\operatorname{Pr}\left[X_{i}\right]=\left(1-\int_{L_{x_{i} x_{i}^{\prime}}} \lambda(x) d x\right)^{n-1} \leq\left(1-\min _{\Psi} \lambda \cdot \delta \cdot r_{n}^{2}\right)^{n-1}$,

where $x_{i}^{\prime}$ is the intersection point of the segment $x_{i}^{\prime} t$ and the circulus of ball $\left(x_{i}, r_{n}\right)$.

From the union bound, we can write ${ }^{3}$

$$
\begin{aligned}
\operatorname{Pr}[X] & \leq n \cdot\left(1-\min _{\Psi} \lambda \cdot \delta \cdot r_{n}^{2}\right)^{n-1} \\
& =e^{\ln n+(n-1) \ln \left(1-\min _{\Psi} \lambda \cdot \delta \cdot r_{n}^{2}\right)} \\
& \leq e^{\ln n-(n-1) \min \Psi \lambda \cdot \delta \cdot r_{n}^{2}} \\
& =e^{(\ln n) \cdot\left(1-\frac{(n-1)}{\ln n} \min _{\Psi} \lambda \cdot \delta \cdot r_{n}^{2}\right)} \\
& =e^{(\ln n) \cdot\left(1-\min _{\Psi} \lambda \cdot \delta \cdot\left(\frac{r_{n}}{\sqrt{\frac{\ln n}{n-1}}}\right)^{2}\right)}
\end{aligned}
$$

where, to write the second inequality, we have used the fact that $\ln (1+x) \leq x$. For successful GEOGREEDY assumption to be hold w.h.p., we want $\operatorname{Pr}[X] \rightarrow 0$ as $n \rightarrow 0$. From the final equation, this can be seen to happen if $\sqrt{\min _{\Psi} \lambda \cdot \delta}$. $r_{n} / \sqrt{(\ln n) / n} \rightarrow \infty$, that is $r_{n}$ is made to decrease strictly slower than $\sqrt{(\ln n) /\left(\min _{\Psi} \lambda \cdot \delta \cdot n\right)}$, with the ratio going to $\infty$ as $n \rightarrow \infty$. From our network model $\operatorname{NPN}\left(n, r_{n}, \alpha\right)$, we have $r_{n}=\sqrt{\left(c_{g} \cdot \ln n\right) / n}$. Therefore, the sufficient condition for successful GEOGREEDY assumption is $c_{g}>\frac{1}{\min _{\Psi} \lambda \cdot \delta}$.

From Theorem 1, in order to satisfy the successful GEOGREEDY assumption, we need to set $c_{g} \geq \frac{0.82}{\min _{\Psi} \lambda}$ for the 2-dimensional case and $c_{g} \geq \frac{0.77}{\min _{\Psi} \lambda}$ for the 3-dimensional case. Obviously, the successful GEOGREEDY assumption is a stronger requirement than connectivity requirement on $G\left(n, r_{n}\right)$. This statement is also supported by Penrose's investigation [36] on the connectivity properties of RGG in the case of arbitrary node distribution (provided that certain technical conditions are satisfied), in which it is shown that $G\left(n, r_{n}\right)$ is connected w.h.p. if and only if $c_{g}>\frac{1}{\pi \cdot \min _{\Psi} \lambda} \approx \frac{0.32}{\min \Psi \lambda}$ for the 2-dimensional case, which is smaller than our $c_{g}$ for successful GEOGREEDY. Gupta and Kumar's classical work [25] shows that in order to maximize the throughput, $r_{n}$ should be as small as possible when the connectivity condition is satisfied. Their conclusion is for the arbitrary routing algorithms. In our case, when GEOGREEDY is used, $r_{n}$ should be as small as possible when the successful GEOGREEDY assumption is satisfied. Therefore we set $c_{g}=\frac{0.82}{\min \Psi \lambda}$ in the following discussion. Note that the corresponding expected number of local neighbors $N e i g \operatorname{Loc}(u)$ is on the order of $\ln n$, which is the same as for the connectivity [36].

A recent result due to Wan and Yi et al. [37] shows that for the 2-dimensional homogeneous $G\left(n, r_{n}\right)$, where $n$ nodes are distributed uniformly and randomly over a unit square, in order

\footnotetext{
${ }^{3}$ For ease of presentation, we neglect edge effects in the following.
} 


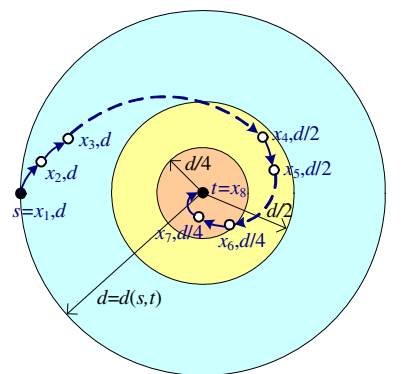

Fig. 3. Approximate GeOGreEDY routing algorithm. Solid curves and dashed curves represent local links and shortcuts, respectively. Notation $x_{4}, d / 2$ means it is the 4-th relay node, with the value of current indicator $d / 2$.

to satisfy the successful GEOGREEDY assumption, $c_{g}$ should be greater than $1 / \delta \approx 0.82$. Obviously, this is a special case of Theorem 1, when the normalized node density is a constant $\lambda(x)=n$, which implies that $\min _{\Psi} \lambda=1$.

\section{Navigability of Nonhomogeneous Poisson NETWORKS}

In this section we will demonstrate the main results of this paper and the corresponding proofs on the navigability of nonhomogeneous Poisson networks. We first present a special routing algorithm used in this section.

An illustrative example is given in Fig.3. If there is no direct link from the source $s$ to the destination $t$, the message (packet) is passed via intermediate nodes as follows. At each stage, the packet carries the address (co-ordinates) of the destination $t$, as well as an indicator in the packet header. The value of the indicator, i.e., $d$, is initialized to $d(s, t)$, the distance between $s$ and $t$. Suppose that the packet is currently at node $u$ and has the indicator value $d>r_{n} \cdot{ }^{4}$ If node $u$ has a shortcut to some node $x_{i} \in A(t, d / 2)$, where the annulus $A(t, d / 2)$ is defined as $A(t, d / 2)=\operatorname{ball}(t, d / 2) \backslash \operatorname{ball}(t, d / 4)$, then $u$ forwards the message to $x_{i}$. If there is more than one such node, the choice can be arbitrary. Otherwise, it forwards the message to one of its local neighbors which is closer to $t$ than itself. When a node $x_{i}$ receives a packet, it updates $d$ with $d / 2$ if $d\left(x_{i}, t\right) \leq d / 2$, and leaves $d$ unchanged otherwise.

In other words, if $u$ can find a shortcut which reduces the distance to the destination by at least a half but by no more than three-quarters, it adopts such a shortcut. Otherwise, it uses a local neighbor to reduce the distance to the destination. In that sense, we call the routing algorithm described above as approximate GEOGREEDY routing algorithm. The reason for considering such an algorithm rather than a GEOGREEDY defined in Def. 5 that minimizes the distance to the destination at each step is to preserve the independence, which greatly simplifies the analysis. Note that if a greedy step from $u$ takes us to $v$ (i.e., of all nodes to which $u$ possesses a shortcut, $v$ is closest to $t$ ), then the conditional law of the point process in the ball $(t, d(t, v))$ will be violated. The fact that there are no shortcuts from $u$ to nodes within this ball biases the probability

${ }^{4}$ If $d \leq r_{n}$, then the node $u$ will have contained $t$ in its local neighbor list and will deliver the packet immediately.

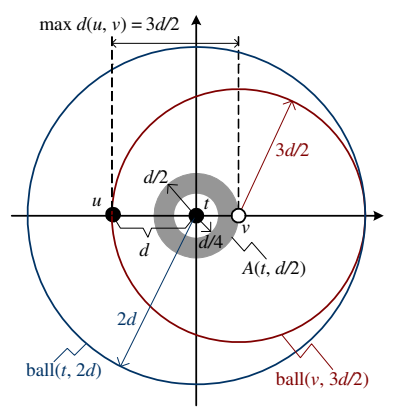

Fig. 4. Calculating the probability of $u$ having a shortcut to one of the nodes in $A(t, d / 2)$.

law and greatly complicates the analysis. Here approximate GEOGREEDY algorithm gets around this problem and has already been proved useful in [22].

\section{A. Navigability of $\operatorname{NPN}\left(n, r_{n}, 1\right)$}

Theorem 2: When $\alpha=1, N P N\left(n, r_{n}, 1\right)$ is a navigable small-world network, i.e., there exists a decentralized routing algorithm, e.g., approximate GEOGREEDY, to route packets between any source-destination pairs chosen from $V \times V$ in $O\left(\log ^{2} n\right)$ hops.

Proof: We concentrate on the 2-d case in the following. The 3-d case can be proven in a similar fashion. Before proceeding to the technical details of the proof, we begin with a brief high-level outline of the proof and some intuition of the analysis. First, we claim that the expected number of hops taken by approximate GEOGREEDY before we reach a node halfway from the source to the destination by utilizing the shortcut is $O(\ln n)$; then we show that after at most $O(\log n)$ such halvings, we will reach the destination w.h.p.

To establish the claim, we first need to calculate the probability of finding a suitable shortcut at each step of the approximate GEOGREEDY. We think of the routing algorithm as proceeding in phases. The value of $d$ is halved at the end of each phase when the packet reaches a node $u$ satisfying the relation $d(u, t) \in(d / 4, d / 2]$ at the first time.

Fig. 4 illustrates the following. Node $u$ is the current packetholder with the destination $t$. Denote by $N_{A}$ the number of nodes in the annulus $A(t, d / 2)$. Obviously

$$
N_{A}=\operatorname{pop}\left(A\left(t, \frac{d}{2}\right)\right)=\operatorname{pop}\left(\operatorname{ball}\left(t, \frac{d}{2}\right) \backslash \operatorname{ball}\left(t, \frac{d}{4}\right)\right) \text {. }
$$

For any $v \in A(t, d / 2)$ and $v \in V$, the distance $d(u, v)$ is bounded above by $3 d / 2$, and thus the probability that a shortcut from $u$ is incident on a particular one of these nodes is bounded below by

$$
\begin{aligned}
\operatorname{Pr}[(v, u)] & \geq a_{n} \cdot(\operatorname{pop}(\operatorname{ball}(v, 3 d / 2)))^{-1} \\
& \geq \frac{a_{n}}{\operatorname{pop}(\operatorname{ball}(t, 2 d))} .
\end{aligned}
$$

Thus, conditional on $N_{A}$, the probability that $u$ has a shortcut to one of the $N_{A}$ nodes in $A(t, d / 2)$ is bounded below by

$$
p\left(d, N_{A}\right) \geq 1-\left(1-\frac{a_{n}}{\operatorname{pop}(\operatorname{ball}(t, 2 d))}\right)^{N_{A}} .
$$


From Eq. (5) and Inequality (7), we obtain

$$
\begin{aligned}
p\left(d, N_{A}\right) & \geq 1-\exp \left(-\frac{N_{A} \cdot a_{n}}{\operatorname{pop}(\operatorname{ball}(t, 2 d))}\right) \\
& =1-\exp \left(-a_{n} \cdot \frac{\operatorname{pop}(\operatorname{ball}(t, d / 2) \backslash \operatorname{ball}(t, d / 4))}{\operatorname{pop}(\operatorname{ball}(t, 2 d))}\right) \\
& \geq a_{n} \cdot \frac{\operatorname{pop}(\operatorname{ball}(t, d / 2) \backslash \operatorname{ball}(t, d / 4))}{\operatorname{pop}(\operatorname{ball}(t, 2 d))} \geq a_{n} .
\end{aligned}
$$

If $u$ does not have such a shortcut, the packet is passed via local neighbors which are successively closer to $t$, and hence the same lower bound on the probability of a shortcut to $A(t, d / 2)$ is satisfied. Consequently, the number of local steps $L_{u}$ until a shortcut is found is bounded above by a geometric random variable with conditional mean $1 / p\left(d, N_{A}\right)$, i.e.,

$$
\operatorname{Pr}\left[L_{u}=k\right]=\left(1-p\left(d, N_{A}\right)\right)^{k-1} \cdot p\left(d, N_{A}\right) .
$$

Therefore, we obtain $\mathbf{E}\left[L_{u}\right] \leq \frac{1}{p\left(d, N_{A}\right)}=\frac{1}{a_{n}}$. Since $a_{n}=$ $\Theta(1 / \ln n)$ (cf. eq. (1) in Section IV-A), we obtain $\mathbf{E}\left[L_{u}\right]=$ $O(\ln n)$. By using the Chernoff bound for a Poisson random variable, it can be shown that $L_{u}=O(\ln n)$ w.h.p.

Note that when $\lambda(x)=\min _{\Psi} \lambda$, the side length $l$ of the smallest square $\mathcal{S}_{n}$ which contains $n$ nodes is at most $O\left(\frac{\sqrt{2 n}}{\min _{\Psi} \lambda}\right)$, which implies that the number of phases is $O(\log n)$ since the initial value of $d$ is at most $O(\sqrt{n})$, and $d$ is halved at the end of each phase.

Therefore, the total number of hops is $O\left(\log ^{2} n\right)$, which completes the proof of the theorem.

Recall the results on homogeneous case, i.e., nodes are uniformly distributed in the space, from works of Kleinberg [13], Franceschetti and Meester [21], Draief and Ganesh [22]. They show that when the shortcut between nodes $u$ and $v$ exists with probability proportional to $d(u, v)^{-2}$ (for the 2-dimensional case), decentralized routing algorithm is efficient. Note that this is a special case of our Theorem 2. In our network model, when nodes are uniformly distributed, i.e., $\lambda(x)=\lambda$ for $\forall x \in \mathcal{S}$, we have

$$
\begin{aligned}
\operatorname{Pr}[(u, v)] & =a_{n} \cdot(\operatorname{pop}(\operatorname{ball}(u, d(u, v))))^{-1} \\
& =\frac{a_{n}}{\lambda \pi d(u, v)^{2}} \propto d(u, v)^{-2} .
\end{aligned}
$$

Therefore, our work can also be applied to homogeneous cases and gives more general condition on the navigability of any geometric network. Our results show that it is the populationdensity based shortcut distribution which determines the navigability of the geometric networks rather than the geographicdistance based shortcut distribution suggested in Kleinberg's work in a more general setting.

\section{B. Innavigability of $\operatorname{NPN}\left(n, r_{n}, \alpha\right)$ When $\alpha \neq 1$}

In this subsection, we show that $\alpha=1$ is also the necessary condition for greedy geographic routing to be efficient.

Theorem 3: Suppose the source $s$ and destination $t$ are chosen uniformly at random from $V \times V$. (a) When $\alpha>1$, the expected number of hops for routing packets between $s$ and $t$ is $\Omega\left(n^{(\alpha-1) /(2 \alpha)}\right)$; (b) When $0 \leq \alpha<1$, the expected number of hops for routing packets between $s$ and $t$ is $\Omega\left(n^{(1-\alpha) / 6}\right)$.

Proof: (a) For any node $u \in V$, we sort all the other nodes in $V$ using the distance to $u$ in the increasing order. Since nodes are distributed as a Poisson point process, no two nodes are of the same distance to $u$ w.h.p. Therefore, we can obtain a sequence $x_{1}^{u}, x_{2}^{u}, \cdots, x_{i}^{u}, \cdots, x_{n-1}^{u}$, where $x_{i}^{u} \in V$ and $x_{i}^{u} \neq u$ for $1 \leq i \leq n-1$ with the property that (8) $d\left(u, x_{i}^{u}\right)<d\left(u, x_{i+1}^{u}\right)$ for $1 \leq i \leq n-2$. Let $v$ be a longrange neighbor of $u$, the probability that $d(u, v)$ is greater than $d\left(u, x_{i}^{u}\right)$ is bounded above by

$$
\sum_{j=i+1}^{n-1} \frac{a_{n}}{j^{\alpha}} \leq a_{n} \cdot \int_{i}^{\infty} x^{-\alpha} d x \leq \frac{a_{n}}{\alpha-1} i^{1-\alpha} .
$$

Since $a_{n}=\Theta(\alpha-1)$ (cf. eq. (1) in Section IV-A), we obtain

$$
\operatorname{Pr}\left[(u, v) \mid d(u, v)>d\left(u, x_{i}^{u}\right)\right]=O\left(i^{1-\alpha}\right) .
$$

For randomly chosen $s$ and $t, d(s, t)>d\left(t, x_{\sqrt{n}}^{t}\right)$ w.h.p. Define $l^{\prime}=n^{(1 / 2)-\beta} \cdot(\log n)^{-1}$. Therefore, the necessary condition for existing a path of length $n^{\beta}$ hops between $s$ and $t$ is that at least one of the hops is a shortcut of length $l^{\prime}$ (in hops) or more. Let $\varepsilon$ be the event that such a shortcut exists. By eq. (9) and the union bound, we obtain:

$$
\operatorname{Pr}[\varepsilon]=O\left(n^{\beta} \cdot\left(\frac{n^{(1 / 2)-\beta}}{\log n}\right)^{1-\alpha}\right) .
$$

It is obvious that this probability tends to 0 as $n \rightarrow \infty$ if $\beta<(\alpha-1) /(2 \alpha)$, which implies that if $\beta<(\alpha-1) /(2 \alpha)$, then the probability of finding a path with fewer than $n^{\beta}$ hops between $s$ and $t$ tends to zero as $n \rightarrow \infty$.

(b) Define $\beta=(1-\alpha) / 6$. Let $U$ denote the set of nodes within distance $d\left(t, x_{n^{\beta}}^{t}\right)$ of $t$. Therefore, $|U|=n^{\beta}+1$. For randomly chosen $s$ and $t$, it is clear that $s$ is not in $U$ w.h.p., and for any $v \in U, d(s, v)>d\left(v, x_{\sqrt{n}}^{v}\right)$ w.h.p.

Suppose now that there is a distributed routing algorithm which can find a path from $s$ to $t$ in fewer than $n^{\beta}$ hops. Denote by $s=x_{0}, x_{1}, \cdots, x_{m}=t$, the sequence of nodes visited by the routing algorithm, with $m<n^{\beta}$. Then there must be a shortcut from at least one of the nodes $x_{0}, x 1, \cdots$ $\cdot, x_{m-1}$ to the node in $U$. We prove this claim by contradiction. Suppose that the current packet holder is $x_{i}^{t}$, then by only exploiting the local link, the next packet holder will at most be $x_{i-\Theta(\ln n)}^{t}$ (the expected number of local neighbors is on the order of $\ln n$, cf. Section IV-B). Therefore, in order to reach a node in $U$, we need at least $\Omega(\sqrt{n} / \ln n)$ local hops, which contradicts the assumption that the routing algorithm only needs $O\left(n^{\beta}\right)$ hops. Let $\varepsilon$ be the event that within $n^{\beta}$ hops, there exists at least one shortcut from $u \notin U$ to the node in $U$. For a node $u \notin U$, the probability that it has a long-range neighbor in $U$ is upper-bounded by $O\left(|U| \cdot a_{n}\right)$. By eq. (1) and the union bound we obtain

$\operatorname{Pr}[\varepsilon]=O\left(|U| \cdot a_{n} \cdot n^{\beta}\right)=O\left(n^{2 \beta} \cdot n^{\alpha-1}\right)=O\left(n^{-\frac{2}{3}(1-\alpha)}\right)$,

which goes to zero as $n \rightarrow \infty$ when $0 \leq \alpha<1$. Therefore, we conclude that the probability of finding a path with fewer than $n^{\beta}$ hops also tends to zero. 
Note that when $\alpha=0$, the shortcuts are formed as an ErdösRényi random graph. More precisely, shortcuts are present between each pair of nodes with probability $p_{n}=\Theta(\ln n)$, independent of all other shortcuts. Theorem 3 (b) shows that $\operatorname{NPN}\left(n, r_{n}, 0\right)$ is innavigable, even though the diameter of $N P N\left(n, r_{n}, 0\right)$ is $O(\log n)$ w.h.p.

The authors of [30], [31] suggest to uniformly and randomly add some wired shortcuts in wireless ad hoc networks in order to increase transport capacity. Even though their results show the significant improvement both theoretically and experimentally, they fail to consider the algorithmic aspect of their scheme. Our results in this paper show that there is no decentralized algorithm that can achieve those benefits from the short paths existing in the network.

\section{CONCLUSION}

In this paper, we extend Kleinberg's model [13] to a more realistic model constructed from a nonhomogeneous Poisson point process, wherein each node is connected to all its neighbors within some fixed radius, as well as possessing random shortcuts to more distant nodes. More importantly, we show that in nonhomogeneous cases, the necessary and efficient condition for greedy geographic routing to be efficient is that the probability of a shortcut being present from node $u$ to $v$ should be inversely proportional to the number of nodes which are closer to $u$ than $v$ is. Note that our model gives the same shortcut probabilities as models in previous work wherein the nodes are distributed uniformly, therefore, our work can also be applied to homogeneous cases and gives more general condition on the navigability of any geometric network. To the best of our knowledge, this is the first work to prove this result for the general or nonhomogeneous continuum setting.

\section{REFERENCES}

[1] S. Milgram, "The small world problem," Psychology Today, vol. 1, pp. 61-67, May 1967.

[2] T. Achacoso and W. Yamamoto, AY's Neuroanatomy of C.elegans of Computation. Boca Raton, FL: CRC Press, 1992.

[3] R. Williams and D. Martinez, "Simple rules yield complex food webs," Nature, vol. 404, no. 4, pp. 180-183, March 2000.

[4] D. Watts and S. Strogatz, "Collective dynamics of small-world networks," Nature, vol. 393, no. 4, pp. 440-442, June 1998.

[5] R. Albert, H. Jeong, and A.-L. Barabasi, "The diameter of the world wide web," Nature, vol. 401, pp. 130-131, Sep. 1999.

[6] J. Aspnes, Z. Diamadi, and G. Shah, "Fault-tolerant routing in peer-topeer systems," in Proc. of PODC 2002, Monterey, CA, July 2002.

[7] G. Manku, M. Naor, and U. Wieder, "Know thy neighbor's neighbor: The power of lookahead in randomized p2p networks," in Proc. of STOC 2004, Chicago, IL, June 2004.

[8] H. Zhang, A. Goel, and R. Govindan, "Using the small-world model to improve freenet performance," in Proc. of IEEE INFOCOM 2002, New York, NY, June 2002.

[9] S. Capkun, L. Buttyan, and J. Hubaux, "Small worlds in security systems: an analysis of the pgp certificate graph," in New Security Paradigms Workshop 2002, Norfolk, VA, Sep. 2002.

[10] B. Bollobás, Random Graphs. Orlando, FL: Academic Press, 1985.

[11] S. Janson, T. Luczak, and A. Rucinski, Random Graphs. New York: John Wiley \& Sons, 2000.

[12] R. Hekmat, Ad-hoc Networks: Fundamental Properties and Network Topologies. New York: Springer, 2006.

[13] J. Kleinberg, "The small-world phenomenon: An algorithmic perspective," in Proc. 32nd ACM Symposium on Theory of Computing, Portland, Oregon, May 2000.

[14] $\frac{-}{2000}$, "Navigation in a small world," Nature, vol. 406, p. 845, August
[15] D. Coppersmith, D. Gamarnik, and M. Sviridenko, "The diameter of a long-range percolation graph," Random Structures and Algorithms, vol. 21, no. 1, pp. 1-13, August 2002.

[16] J. Kleinberg, "Complex networks and decentralized search algorithms," in Proc. of the International Congress of Mathematicians (ICM), Madrid, Spain, August 2006.

[17] L. Barrière, P. Fraigniaud, E. Kranakis, and D. Krizanc, "Efficient routing in networks with long range contacts," in Proc. of the 15th International Symposium on Distributed Computing (DISC 01), Lisboa, Portugal, Oct. 2001.

[18] C. Martel and V. Nguyen, "Analyzing kleinberg's (and other) smallworld models," in Proc. of PODC 2004, Newfoundland, Canada, July 2004.

[19] P. Fraigniaud, C. Gavoille, and C. Paul, "Eclecticism shrinks even small worlds," in Proc. of PODC 2004, Newfoundland, Canada, July 2004.

[20] E. Lebhar and N. Schabanel, "Almost optimal decentralized routing in long-range contact networks," in Proc. of ICALP 2004, Turku, Finland, July 2004 .

[21] M. Franceschetti and R. Meester, "Navigation in small-world networks: a scale-free continuum model," Journal of Applied Probability, vol. 43, no. 4, pp. 1173-1180, Dec. 2006.

[22] M. Draief and A. Ganesh, "Efficient routing in poisson small-world networks," Journal of Applied Probability, vol. 43, no. 3, pp. 678-686, June 2006.

[23] D. Liben-Nowell, J. Novak, R. Kumar, P. Raghavan, and A. Tomkins, "Geographic routing in social networks," Proceedings of the National Academy of Sciences, vol. 102, no. 33, pp. 11 623-11 628, August 2005.

[24] J. Kleinberg, "Small-world phenomena and the dynamics of information," Advances in Neural Information Processing Systems, vol. 14, 2001.

[25] P. Gupta and P. R. Kumar, "The capacity of wireless networks," IEEE Transactions on Information Theory, vol. 46, no. 2, pp. 388-404, March 2000.

[26] M. Penrose, Random Geometric Graphs. Oxford: Oxford University Press, 2003.

[27] B. Liu, Z. Liu, and D. Towsley, "On the capacity of hybrid wireless networks," in Proc. of InfoCom 2003, San Francisco, CA, April 2003.

[28] S. Kulkarni and P. Viswanath, "Throughput scaling in heterogeneous networks," in Proc. of IEEE ISIT 2003, Kanagawa, Japan, June 2003.

[29] A. Helmy, "Small worlds in wireless networks," IEEE Communications Letters, vol. 7, no. 10, pp. 490-492, Oct. 2003.

[30] A. Reznik, S. Kulkarni, and S. Verdú, "Scaling laws in random heterogeneous networks," in Proc. of ISIT 2004, Chicago, USA, June 2004.

[31] - "A "small world" approach to heterogeneous networks," Communications in Information and Systems, vol. 3, no. 4, pp. 325-348, September 2004

[32] G. Sharma, N. Shroff, and R. Mazumdar, "Hybrid sensor and mesh networks: Paradigms for fair and energy efficient communication," in Proc. of WiMesh 2006, Reston, Virginia, September 2006.

[33] R. Chitradurga and A. Helmy, "Analysis of wired shortcuts in wireless sensor networks," in Proc. of IEEE/ACS International Conference on Pervasive Services, Beirut, Lebanon, July 2004.

[34] G. Sharma and R. Mazumdar, "Hybrid sensor networks: A small world," in Proc. of ACM MobiHoc 2005, Urbana-Champaign, IL, May 2005.

[35] D. Stoyan, W. Kendall, and J. Mecke, Stochastic Geometry and its Applications, 2nd ed. New York: Wiley, 1995.

[36] M. Penrose, "A strong law for the longest edge of the minimal spanning tree," The Annals of Probability, vol. 27, no. 1, pp. 246-260, Jan. 1999.

[37] P.-J. Wan, C.-W. Yi, F. Yao, and X. Jia, "Asymptotic critical transmission radius for greedy forward routing in wireless ad hoc networks," in Proc. of ACM MobiHoc 2006, Florence, Italy, May 2006. 